\title{
The Influencing Factors of Acceptance of Disablility in Spinal Cord Injured Patients
}

\section{Tuenchai Attawong, BSc, MS}

Department of Rehabilitation Medicine Faculty of Medicine

Chiang Mai University

Chiang Mai, Thailand

\section{Apichana Kovindha, MD, FRC Physiatr T, FISCoS}

Department of Rehabilitation Medicine

Faculty of Medicine

Chiang Mai University

Chiang Mai, Thailand

Address for correspondence:

Tuenchai Attawong, BSc, MS

Department of Rehabilitation Medicine

Faculty of Medicine

Chiang Mai University

Chiang Mai 50200, Thailand

Email: tattawon@mail.med.cmu.ac.th

Received, November 19, 2004

Accepted, December 28, 2004
The objective of this study was to examine the psychological factors related to acceptance of disability and to compare the correlated factors with duration of spinal cord injury (SCI). Demographic data, the Acceptance of Disability Scale, the Hospital Anxiety and Depression Scale and the General Perceived Self-Efficacy Scale were analyzed to assess correlation with acceptance of disability of SCI persons attending the outpatient Clinic or admitted to the Rehabilitation Ward, Maharaj Nakorn Chiang Mai Hospital, Chiang Mai between April and May 2004.

Sixty-one SCI persons had completed a self-report measure with mean age of 36.6 years and mean duration after SCI of 48.5 months. They were divided into 2 groups-those with injury more than 6 months and those within last 6 months after injury, and were compared in regard to acceptance of disability. Self-efficacy had positive correlation while depression and anxiety were negatively correlated with acceptance of disability $(\mathrm{r}=.511,-.488,-.456, \mathrm{p}<0.01)$. Sex, educational level, status, severity, duration after SCI and age had no significant correlation with acceptance of disability. In addition, SCI persons who had duration after SCI of more than 6 months reported more acceptance of disability than those being SCI for less than 6 months, though not to the point of reaching the statistical significance.

In conclusion, this study shows that self-efficacy and emotional status are correlated with acceptance of disability. SCI persons will be able to adjust to their disability more easily if social learning and emotional support are emphasized.

Key Words: Disability, psychological factors, spinal cord injury
A cceptance of disability in SCI persons is desirable. SCI persons who adjust well to unexpected events, generally lead healthy, active and happy lives after injury but those with negative acceptance have a harder time accepting their changes in appearance and have difficulty in coping. There are many factors, including age, sex, severity, marital status, educational level, duration of injury, emotional response, social support, coping styles and self-perception, that influence the acceptance of disability. 1,6,8,9,5

However factors that correlated with acceptance of disability in Thai SCI patients have never been studied. Therefore, this study was undertaken to examine such factors and to compare them with duration after injury.

\section{Materials and Methods}

\section{Materials}

Demographic data containing age, educational level, marital status, sex, severity of disability and duration of injury were obtained. Three self-administered questionnaires were used and completed by the patients. These were as follows:

- The Acceptance of Disability Scale (AD Scale) developed by Linkowski (1971) which has been widely regarded to have content and constructional validity. The Scale was translated into Thai using a back translation method and tested on 97 Thai physically disabled persons. The Conbrach Alpha internal consistency of the Scale was .91. The AD Scale has 50 items and describes self-attitudes towards disability and is based on the concept of acceptance of loss.

- The Thai version of Hospital Anxiety and Depression Scale (HADS) is a 14 item questionnaire that measures anxiety and depression symptoms.

- The Thai version of General Perceived Self-Efficacy Scale (GSES) is a 10 item questionnaire that measures individual beliefs about his or her ability to cope with stressful life events in various domains of 


\begin{tabular}{lllll}
\hline \multicolumn{1}{c}{ Factors } & Minimum & Maximum & Mean & SD \\
\hline Age (years) & 18 & 73 & 36.6 & 13.3 \\
\hline Duration after injury (months) & 1 & 380 & 48.5 & 72.7 \\
\hline & \multicolumn{3}{c}{ Number } & $\%$ \\
\hline Gender & & 77 \\
$\quad$ Male & 47 & 23 \\
Female & 14 & 52.5 \\
\hline Marital status & & 37.7 \\
Single & 32 & 8.2 \\
Married & 23 & 1.6 \\
Divorced & 5 & 1.6 \\
Widowed & 1 & 41.0 \\
\hline Educational level & & 39.4 \\
No education & 1 & 18.0 \\
Primary level & 25 & \\
Secondary level & 24 & 23.0 \\
Undergraduate level & 11 & 37.6 \\
\hline Severity of impairment/disability & 14.4 \\
Paraplegia, incomplete & 14 & 23.0 \\
Paraplegia, complete & 10 & \\
Tetraplegia, incomplete & & \\
Tetraplegia, omplete & & \\
\hline
\end{tabular}

Table 1: Demographic and medical variables in patients with spinal cord injuries. $(N=61)$

human functioning and the performance in difficult tasks.

\section{Procedure and Participants}

The study was reviewed and approved by the Ethical Committee at the Faculty of Medicine, Chiang Mai University. Each participant was informed of the voluntary nature of participation and signed informed consent forms before completing the three questionnaires. Sixty-one SCI patients were recruited from the Rehabilitation Ward I and the Outpatient Clinic, Department of Rehabilitation Medicine, Maharaj Nakorn Chiang Mai Hospital, Faculty of Medicine, Chiang Mai University between April and May, 2004. The selection criteria for inclusion were a traumatic SCI, age $>18$ years and good cognitive function. The participants who reported that they had serious co-morbidity and psychiatric problems were excluded.

\section{Statistics}

The SPSS version 10.0 was used for analysis and four sets of statistical analyses were performed:
- Descriptive statistics were generated for demographic variables and duration of injury.

- Pearson correlations were calculated between acceptance of disability score, anxiety and depression, self-efficacy, and variables in demographic data.

- Multiple step wise regression was used to predict the variables that correlated with acceptance of disability.

- Unpaired t-test was used to compare the correlated factors between acute and chronic SCI groups (less than and more than 6 months respectively)

\section{Results}

\section{Demographic and Medical Characteristics of Sample}

There were 61 SCI persons: 47 men and 14 women. Mean age was $36.6+13.3$ years and mean duration after injury was $48.5+72.7$ months. More than $50 \%$ were single and $41 \%$ had primary education. The majority of SCI $(60.6 \%)$ had complete lesion (Table 1). 


\begin{tabular}{lcccc}
\hline Variables & $\begin{array}{c}\text { Acceptance } \\
\text { of disability }\end{array}$ & Self-efficacy & Depression & Anxiety \\
\hline Age & .060 & $.282^{*}$ & -.016 & -.059 \\
Duration of injury & .170 & .058 & -.013 & -.127 \\
Gender & .094 & .133 & .301 & .206 \\
Marital status & .050 & -.400 & .208 & .143 \\
Educational level & .086 & -.106 & .100 & .051 \\
Severity of disability & .145 & .099 & .049 & .031 \\
Self-efficacy & $.511^{* *}$ & 1 & $-.387^{* *}$ & $-.359^{* *}$ \\
Depression & $-.488^{* *}$ & - & 1 & $.809^{* *}$ \\
Anxiety & $-.456^{* *}$ & - & - & 1 \\
\hline$* 0<0.01, " \mathrm{p}<0.05$ & & & &
\end{tabular}

Table 2. Correlation between acceptance of disability, demographic variables, self-efficacy, depression and anxiety.

\section{Correlations between Acceptance of Disability and Demographic Variables, Self-efficacy, Depression and Anxiety}

Table 2 summarizes the overall variables correlated with acceptance of disability in SCI. A significantly high positive correlation was found between acceptance of disability and self-efficacy $(\mathrm{r}=.511, \mathrm{p}<0.01)$, whereas there was a significant negative correlation between acceptance of disability and symptoms of depression and anxiety $(\mathrm{r}=-.488,-.456, \mathrm{p}<0.01)$. There was no significant correlation between acceptance of disability and age, duration of injury, sex, marital status, educational level and severity of impairment/disability. There were interrelations among age, self-efficacy, depression and anxiety. Self-efficacy had positive correlation with age $(\mathrm{r}=.282, \mathrm{p}<.05)$ but had negative correlation with depression and anxiety $(\mathrm{r}=-.387, \mathrm{r}=-.359, \mathrm{p}<.01)$, whereas depression was positively correlated with anxiety $(\mathrm{r}=.809, \mathrm{p}<.01)$.

A multiple stepwise regression analysis was conducted to examine the relationship between the acceptance of disability and self-efficacy, depression and anxiety. It was found that only high self-efficacy and low depression symptoms were statistically significant predictors of acceptance of disability in SCI for $36 \%\left(\mathrm{R}^{2}=.360, \mathrm{~F}=16.329\right.$, $\mathrm{p}<0.01)$. The beta weights for the self-efficacy and for depression were .378 and -.342 , respectively $(\mathrm{p}<0.01)$.

\section{Comparison of Correlation Variables with Acceptance of Disability and Duration of Injury}

Sixty-one SCI persons were divided into 2 groups: 31 acute and 30 chronic. This study found no difference in mean scores of acceptance of disability in the 2 groups (acute 174.81 and chronic 181.30, $\mathrm{p}=.430$ ). Moreover there were no significant differences between acute and chronic groups in regard to self-efficacy, depression and anxiety (Table 3).

\section{Discussion}

According to this study, self-efficacy and symptoms of depression and anxiety were significantly correlated with acceptance of disability of SCI persons. High self-efficacy was positively correlated with acceptance of disability. It is the most powerful predictor of acceptance of disability. Therefore self-efficacy reflects one's power of managing the burden of disability and this power comes from oneself. It is more likely that SCI persons use it to control behavior and attitude, and it can lead them to self-realization, ${ }^{10}$ and to select challenging tasks and perform them successfully.

Low level of depression and anxiety was negatively correlated with acceptance of disability, suggesting that SCI persons may have negative views of themselves, the environment and future. Many SCI persons continue to hold onto irrational beliefs after their injury, such as "no one will accept or respect disabled persons and their lives are less important than those of non-disabled persons". These beliefs may lead to emotional responses such as sadness, confusion, anger, anxiety and fear. According to this study, most SCI persons still have sustained depression and anxiety as time passed. This finding supports the studies of Kennedy and Roger ${ }^{6}$ and Craig, et al., ${ }^{\text {that }}$ SCI persons had a high level of depression and anxiety in the acute and the rehabilitation phases, and depression still persists after discharge. In addition, SCI persons who have coped well in life and never experienced depression or other severe emotional responses may ensure appropriate adjustment to disability.

Concerning the demographic variables including age, marital status, educational level and gender, there was no significant correlation with acceptance of disability. This finding does not support previous studies reporting that younger SCI persons seem to have better acceptance of disability. This may be due to their flexible attitude to life and perception of their disability and limitations. ${ }^{3,7}$ Regarding acceptance of reality, it usually takes time for newly SCI persons to accept disability. If they were injured as a child, they easily develop a self-concept of future and have more satisfaction in life and working arrangements. According to the studies of El Ghatit and Hanson, and Crewe and Krause, females were found to cope better and more easily accept disability than males. ${ }^{2,4}$ In addition, marital status and high education are important predictors of adjustment. ${ }^{4,2}$ In this 


\begin{tabular}{|c|c|c|c|c|}
\hline \multirow[b]{2}{*}{ Variables } & \multirow[b]{2}{*}{$\begin{array}{l}\text { Total } \\
(\mathrm{N}=61)\end{array}$} & \multicolumn{3}{|c|}{ Duration of injury } \\
\hline & & $\begin{array}{c}\text { Acute } \\
\text { Less than } 6 \text { months } \\
(\mathrm{N}-31)\end{array}$ & $\begin{array}{c}\text { Chronic } \\
\text { More than } 6 \text { months } \\
(\mathrm{N}-30)\end{array}$ & $\begin{array}{c}\text { P } \\
\text { value }\end{array}$ \\
\hline Self-efficacy & $31.52+4.28$ & $31.52+4.90$ & $31.53+3.63$ & .988 \\
\hline Depression & $4.54+3.45$ & $4.80+4.00$ & $4.27+2.82$ & .546 \\
\hline Anxiety & $5.00+3.24$ & $5.16+3.60$ & $4.8+2.88$ & .696 \\
\hline
\end{tabular}

Table 3: Comparison of mean score of correlation variables with acceptance of disability and duration of injury between acute and chronic SCI group

study severity of disability, however, was not found to be correlated with acceptance of disability. This supports the finding that severity of disability was not important in accepting life with disability. ${ }^{11}$ However, age had positive correlation with self-efficacy. Suggesting that adults with SCI, who have more experience of success in daily tasks and cope well, usually perceive their ability and be happy in life with disability.

From this study, we found that the key components of accepting disability are emotional status and socialcognition. In addition, most SCI persons required formal support such as social support from family, disabled peers, friends and the community. These are essential for disabled persons throughout their life.

Several limitations in this study should be noted such as small sample size, mainly men, wide range of duration after injury. Lastly, the study did not cover other variables such as social support, employment outcome, coping skills and life satisfaction that may affect acceptance of disability. For further research, the above-mentioned variables should be investigated for future clinical implication.

\section{Conclusions}

This study reveals a better understanding of factors that are related to acceptance of disability in Thai SCI persons. It is important that the rehabilitation team should have knowledge and understanding of their patients' perception during hospitalization and after discharge to the community. Emotional support is important during periods of sadness. The supportive strengthening and physical skill training can help SCI persons maintain hope and motivation, and realize their gained capacity after the rehabilitation program. In some cases, formal counseling and formal psychological support is required. In addition, enhancement of self-efficacy, including providing skills for self-management in activities of daily living, problem-solving strategies and gaining selfconfidence should be emphasized in the rehabilitation program.

\section{References}

1. Craig AR, Hancock KM, Dickson HG: A longitudinal investigation into anxiety and depression in the first 2 years following a spinal cord injury. Paraplegia 32:675-679, 1994.

2. Crewe NM, Krause JS: Marital relationships and spinal cord injury. Arch Phys Med Rehabil 69:435438, 1988.

3. Dijkers M: Quality of life after spinal cord injury: A metanalysis of disablement components. Spinal Cord 35:829-840, 1997.

4. El Ghatit AZ, Hanson RW: Education and training levels and employment of spinal cord injured patients. Arch Phys Med Rehabil 60:405-406, 1979.

5. Hampton NZ: Disability status, perceived health, social support, self-efficacy, and quality of life among people with spinal cord injury in the People's Republic of China. Int J Rehabil Res 24:69-71, 2001.

6. Kennedy P, Lowe R, Grey N, et al: Traumatic spinal cord injury and psychological impact: Cross- sectional analysis of coping strategies. Br J Clin Psych 34:627-639, 1995.

7. Kennedy P, Roger BA: Anxiety and depression after spinal cord injury: A longitudinal analysis. Arch Phys Med Rehabil 81:932-937, 2000.

8. Krause JS: Adjustment after spinal cord injury: A 9 years longitudinal study. Arch Phys Med Rehabil 78:651-657, 1997.

9. Krause JS, Kemp B, Coker J: Depression after spinal cord injury: Relation to gender, ethnicity, aging, and socioeconomic indicators. Arch Phys Med Rehabil 8: 1099-1109, 2000.

10. Nosek MA, Hughes RB: Psychological aspects of sense of self in women with physical disability. $\mathbf{J}$ Rehabil 67:20-25, 2001.

11. Woodrich F, Patterson JB: Variables related to acceptance of disability in persons with spinal cord injury. J Rehabil 49:26-30, 1983 